\title{
Sub-stabilizability and super-stabilizability for bivariate means
}

\author{
Mustapha Raïssouli ${ }^{1,2^{*}}$ and József Sándor ${ }^{3}$
}

"Correspondence:
raissouli_10@hotmail.com
'Department of Mathematics,
Science Faculty, Taibah University,
P.O. Box 30097, Al Madinah Al
Munawwarah, 41477, Kingdom of
Saudi Arabia
2Department of Mathematics,
Science Faculty, Moulay Ismail
University, Meknes, Morocco
Full list of author information is
available at the end of the article

available at the end of the article

\begin{abstract}
The stability and stabilizability concepts for means in two variables have been introduced in (Raïssouli in Appl. Math. E-Notes 11:159-174, 2011). It has been proved that the arithmetic, geometric, and harmonic means are stable, while the logarithmic and identric means are stabilizable. In the present paper, we introduce new concepts, the so-called sub-stabilizability and super-stabilizability, and we apply them to some standard means.
\end{abstract}

MSC: $26 \mathrm{E} 60$

Keywords: means; stable means; stabilizable means; sub-stabilizable means; super-stabilizable means; mean-inequalities

\section{Introduction}

In this section, we recall some basic notions about means in two variables that will be needed later. Throughout the following, we understand by a (bivariate) mean a binary map $m$ between positive real numbers satisfying the following statement:

$$
\forall a, b>0, \quad \min (a, b) \leq m(a, b) \leq \max (a, b) .
$$

Every mean satisfies $m(a, a)=a$ for each $a>0$. The maps $(a, b) \longmapsto \min (a, b)$ and $(a, b) \longmapsto \max (a, b)$ are (trivial) means, which will be denoted by min and max, respectively. The standard examples of means are given in the following (see [1] for instance and the related references cited therein):

$$
\begin{aligned}
& A:=A(a, b)=\frac{a+b}{2} ; \quad G:=G(a, b)=\sqrt{a b} ; \quad H:=H(a, b)=\frac{2 a b}{a+b} ; \\
& L:=L(a, b)=\frac{b-a}{\ln b-\ln a}, \quad L(a, a)=a ; \\
& I:=I(a, b)=e^{-1}\left(\frac{b^{b}}{a^{a}}\right)^{1 /(b-a)}, \quad I(a, a)=a
\end{aligned}
$$

and are known as the arithmetic, geometric, harmonic, logarithmic, and identric means, respectively.

There are more means of interest known in the literature. For instance, the following:

$$
P:=P(a, b)=\frac{b-a}{4 \arctan \sqrt{b / a}-\pi}=\frac{b-a}{2 \arcsin \frac{b-a}{b+a}}, \quad P(a, a)=a ;
$$

@2014 Raïssouli and Sándor; licensee Springer. This is an Open Access article distributed under the terms of the Creative Commons Attribution License (http://creativecommons.org/licenses/by/2.0), which permits unrestricted use, distribution, and reproduction in any medium, provided the original work is properly cited. 


$$
\begin{aligned}
& T:=T(a, b)=\frac{b-a}{2 \arctan \frac{b-a}{b+a}}, \quad T(a, a)=a ; \\
& M:=M(a, b)=\frac{b-a}{2 \operatorname{arcsinh} \frac{b-a}{b+a}}, \quad M(a, a)=a ;
\end{aligned}
$$

are known as the first Seiffert mean [2], the second Seiffert mean [3] and the NeumanSándor mean [4], respectively.

A mean $m$ is symmetric if $m(a, b)=m(b, a)$ for all $a, b>0$, and monotone if $(a, b) \longmapsto$ $m(a, b)$ is increasing in $a$ and in $b$, that is, if $a_{1} \leq a_{2}$ (resp. $\left.b_{1} \leq b_{2}\right)$ then $m\left(a_{1}, b\right) \leq m\left(a_{2}, b\right)$ (resp. $\left.m\left(a, b_{1}\right) \leq m\left(a, b_{2}\right)\right)$. For more details as regards monotone means, see [5].

For two means $m_{1}$ and $m_{2}$ we write $m_{1} \leq m_{2}$ if and only if $m_{1}(a, b) \leq m_{2}(a, b)$ for every $a, b>0$ and, $m_{1}<m_{2}$ if and only if $m_{1}(a, b)<m_{2}(a, b)$ for all $a, b>0$ with $a \neq b$. Two means $m_{1}$ and $m_{2}$ are comparable if $m_{1} \leq m_{2}$ or $m_{2} \leq m_{1}$, and we say that $m$ is between two comparable means $m_{1}$ and $m_{2}$ if $\inf \left(m_{1}, m_{2}\right) \leq m \leq \sup \left(m_{1}, m_{2}\right)$. If the above inequalities are strict then we say that $m$ is strictly between $m_{1}$ and $m_{2}$. The above means are all comparable with the well-known chain of inequalities

$$
\min <H<G<L<P<I<A<M<T<\max .
$$

For a given mean $m$, we set $m^{*}(a, b)=\left(m\left(a^{-1}, b^{-1}\right)\right)^{-1}$, and it is easy to see that $m^{*}$ is also a mean, called the dual mean of $m$. Every mean $m$ satisfies $m^{* *}:=\left(m^{*}\right)^{*}=m$, and if $m_{1}$ and $m_{2}$ are two means such that $m_{1}<m_{2}$ then $m_{1}^{*}>m_{2}^{*}$. Further, the arithmetic and harmonic means are mutually dual (i.e. $A^{*}=H, H^{*}=A$ ) and the geometric mean is self-dual (i.e. $\left.G^{*}=G\right)$.

Let $p$ be a real number. The next means are of interest.

- The power (binomial) mean:

$$
\left\{\begin{array}{l}
B_{p}:=B_{p}(a, b):=G_{p, 0}(a, b)=\left(\frac{a^{p}+b^{p}}{2}\right)^{1 / p}, \\
B_{-1}=H, \quad B_{0}=G, \quad B_{1}=A, \quad B_{2}:=Q .
\end{array}\right.
$$

- The power logarithmic mean:

$$
\left\{\begin{array}{l}
L_{p}:=L_{p}(a, b)=\left(\frac{a^{p}-b^{p}}{p(\ln a-\ln b)}\right)^{1 / p}, \quad L_{p}(a, a)=a, \\
L_{-1}=L^{*}, \quad L_{0}=G, \quad L_{1}=L, \quad L_{2}=(A L)^{1 / 2} .
\end{array}\right.
$$

We end this section by recalling the next result which will be needed in the sequel.

Theorem 1.1 The following mean-inequalities hold:

$$
L_{2}<P<B_{2 / 3}, \quad L_{4}<M<B_{4 / 3}, \quad L_{5}<T<B_{5 / 3} .
$$

Further these inequalities are the best possible i.e. $L_{2}, L_{4}, L_{5}$ are the best power logarithmic means lower bounds of $P, M, T$, while $B_{2 / 3}, B_{4 / 3}, B_{5 / 3}$ are the best power (binomial) means upper bounds of $P, M, T$, respectively. Otherwise, there is no $p>0$ such that $P, M$ or $T$ is strictly less that $L_{p}$.

For some details as regards the above theorem, we refer the reader to [6-10]. 


\section{Needed tools}

For the sake of simplicity for the reader, we recall here more basic notions and results that will be needed in the sequel, see [11] for more details. We begin by the next definition.

Definition 2.1 Let $m_{1}, m_{2}$, and $m_{3}$ be three given symmetric means. For all $a, b>0$, define

$$
\mathcal{R}\left(m_{1}, m_{2}, m_{3}\right)(a, b)=m_{1}\left(m_{2}\left(a, m_{3}(a, b)\right), m_{2}\left(m_{3}(a, b), b\right)\right),
$$

called the resultant mean-map of $m_{1}, m_{2}$, and $m_{3}$.

For the computation of $\mathcal{R}\left(m_{1}, m_{2}, m_{3}\right)$ when $m_{1}, m_{2}, m_{3}$ belong to the set of the above standard means, some examples can be found in [11-14]. Here we state another example which will be of interest.

Example 2.1 It is not hard to verify that

$$
\mathcal{R}(A, I, G)=e^{-1}\left(\frac{A G+G^{2}}{2}\right)^{1 / 2} \exp \frac{A+G}{2 L} .
$$

A study investigating the elementary properties of the resultant mean-map has been stated in [11]. In particular, if $m_{1}, m_{2}$, and $m_{3}$ are three symmetric monotone means then the map $(a, b) \longmapsto \mathcal{R}\left(m_{1}, m_{2}, m_{3}\right)(a, b)$ defines a mean, where we have the relationship

$$
\left(\mathcal{R}\left(m_{1}, m_{2}, m_{3}\right)\right)^{*}=\mathcal{R}\left(m_{1}^{*}, m_{2}^{*}, m_{3}^{*}\right) .
$$

We also recall the next result, see [13].

Theorem 2.1 Let $m_{1}, m_{1}^{\prime}, m_{2}, m_{2}^{\prime}, m_{3}$, and $m_{3}^{\prime}$ be strict symmetric monotone means such that

$$
m_{1} \leq m_{1}^{\prime}, \quad m_{2} \leq m_{2}^{\prime} \quad \text { and } \quad m_{3} \leq m_{3}^{\prime} .
$$

Then we have

$$
\mathcal{R}\left(m_{1}, m_{2}, m_{3}\right) \leq \mathcal{R}\left(m_{1}^{\prime}, m_{2}^{\prime}, m_{3}^{\prime}\right)
$$

If moreover there exists $i=1,2,3$ such that $m_{i}<m_{i}^{\prime}$, then one has

$$
\mathcal{R}\left(m_{1}, m_{2}, m_{3}\right)<\mathcal{R}\left(m_{1}^{\prime}, m_{2}^{\prime}, m_{3}^{\prime}\right) .
$$

As already proved [11-13], the resultant mean-map's importance stems from the fact that it is a tool for introducing the stability and stabilizability concepts, which we recall in the following.

Definition 2.2 A symmetric mean $m$ is said to be:

(a) Stable if $\mathcal{R}(m, m, m)=m$.

(b) Stabilizable if there exist two nontrivial stable means $m_{1}$ and $m_{2}$ satisfying the relation $\mathcal{R}\left(m_{1}, m, m_{2}\right)=m$. We then say that $m$ is $\left(m_{1}, m_{2}\right)$-stabilizable. 
A developed study about the stability and stabilizability of the standard means was presented in [11]. In particular the next result has been proved there.

Theorem 2.2 With the above, the following assertions are met:

(1) The power binomial mean $B_{p}$ is stable for all real number $p$. In particular, the arithmetic, geometric, and harmonic means $A, G$, and $H$ are stable.

(2) The power logarithmic mean $L_{p}$ is $\left(B_{p}, G\right)$-stabilizable for all real number $p$.

(3) The logarithmic mean $L$ is $(H, A)$-stabilizable and $(A, G)$-stabilizable while the identric mean I is $(G, A)$-stabilizable.

Remark 2.1 The symmetry character of the above involved mean is, by definition, taken as essential hypothesis. In fact, if we attempt to extend the above concepts to non-symmetric means by keeping the same definitions (Definition 2.1 and Definition 2.2), the simple means $m=A_{1 / 3}, G_{1 / 3}$, with $A_{1 / 3}(a, b)=(1 / 3) a+(2 / 3) b, G_{1 / 3}(a, b)=a^{1 / 3} b^{2 / 3}$, do not satisfy $\mathcal{R}(m, m, m)=m$. In another way, the definition of $\mathcal{R}$, together with that related to the stability and stabilizability concepts, is not exactly the same as above, but must be investigated for non-symmetric means. We leave the details as regards the latter point to a later time.

The next definition is also needed here [13].

Definition 2.3 Let $m_{1}$ and $m_{2}$ be two symmetric means. The tensor product of $m_{1}$ and $m_{2}$ is the map, denoted $m_{1} \otimes m_{2}$, defined by

$$
\forall a, b, c, d>0, \quad m_{1} \otimes m_{2}(a, b, c, d)=m_{1}\left(m_{2}(a, b), m_{2}(c, d)\right) .
$$

A symmetric mean $m$ will be called cross mean if the map $m^{\otimes 2}:=m \otimes m$ is symmetric in its four variables.

It is proved in [11] that every cross mean is stable. The reverse of the latter assertion is still an open problem. Otherwise, it is conjectured [13] that the first Seiffert mean $P$ is not stabilizable and such a problem is also still open. We also conjecture here that the second Seiffert mean and the Neuman-Sándor mean are not stabilizable either.

The next result needed here has also been proved in [14].

Theorem 2.3 Let $m_{1}$ and $m_{2}$ be two nontrivial stable symmetric monotone means such that $m_{1} \leq m_{2}$ (resp. $\left.m_{2} \leq m_{1}\right)$. Assume that $m_{1}$ is moreover a cross mean. Then there exists one and only one $\left(m_{1}, m_{2}\right)$-stabilizable mean $m$ such that $m_{1} \leq m \leq m_{2}\left(\right.$ resp. $m_{2} \leq$ $\left.m \leq m_{1}\right)$.

Recently, Raïssouli and Sándor [5] introduced a mean-transformation defined in the following way: for a given mean $m$ (symmetric or not) they set

$$
m^{\pi}(a, b)=\prod_{n=1}^{\infty} m\left(a^{1 / 2^{n}}, b^{1 / 2^{n}}\right)
$$

This allowed them to construct a lot of new means and to obtain good relationships between some standard means. In particular, they obtained $G^{\pi}=G, A^{\pi}=L, S^{\pi}=I, C^{\pi}=A$ 
and $B_{p}^{\pi}=L_{p}$ for every real number $p$, where $S$ and $C$ refer, respectively, to the weighted geometric mean and contra-harmonic mean defined by

$$
S:=S(a, b)=\left(a^{a} b^{b}\right)^{1 /(a+b)}, \quad C:=C(a, b)=\frac{a^{2}+b^{2}}{a+b} .
$$

\section{Two special subsets of means}

Let $\mathcal{M}_{s}$ be the set of all symmetric means. For fixed $m_{1}, m_{2} \in \mathcal{M}_{s}$, we set

$$
\begin{aligned}
& \mathcal{E}^{-}\left(m_{1}, m_{2}\right)=\left\{m \in \mathcal{M}_{s}, \mathcal{R}\left(m_{1}, m, m_{2}\right) \leq m\right\}, \\
& \mathcal{E}^{+}\left(m_{1}, m_{2}\right)=\left\{m \in \mathcal{M}_{s}, m \leq \mathcal{R}\left(m_{1}, m, m_{2}\right)\right\} .
\end{aligned}
$$

It is clear that $\max \in \mathcal{E}^{-}\left(m_{1}, m_{2}\right)$ and $\min \in \mathcal{E}^{+}\left(m_{1}, m_{2}\right)$, that is, these sets are nonempty. Moreover, by equation (2.1) the relationship

$$
m \in \mathcal{E}^{-}\left(m_{1}, m_{2}\right) \quad \Longleftrightarrow \quad m^{*} \in \mathcal{E}^{+}\left(m_{1}^{*}, m_{2}^{*}\right)
$$

is obvious. By virtue of this equivalence, it will be sufficient to study the properties of one the sets $\mathcal{E}^{-}\left(m_{1}, m_{2}\right)$ and $\mathcal{E}^{+}\left(m_{1}, m_{2}\right)$ and to deduce that of the other by duality.

Example 3.1 With the help of Theorem 2.1, it is simple to see that $G<\mathcal{R}(G, G, A)$ and $A>\mathcal{R}(G, A, A)$. So $G \in \mathcal{E}^{+}(G, A)$ and $A \in \mathcal{E}^{-}(G, A)$. We can also verify that $T \in \mathcal{E}^{-}(A, G)$ and $M \in \mathcal{E}^{-}(A, G)$. Other more interesting examples will be seen later.

The next result is of interest.

Proposition 3.1 Let $m_{1}, m_{2}$ be two nontrivial monotone (symmetric) stable means where $m_{1}$ is a cross mean. Then the intersection between $\mathcal{E}^{-}\left(m_{1}, m_{2}\right)$ and $\mathcal{E}^{+}\left(m_{1}, m_{2}\right)$ is reduced to the unique mean $m$ which is the $\left(m_{1}, m_{2}\right)$-stabilizable mean.

Proof Following Theorem 2.3, let $m$ be the unique $\left(m_{1}, m_{2}\right)$-stabilizable mean. Then $\mathcal{R}\left(m_{1}, m, m_{2}\right)=m$ and so $m \in \mathcal{E}^{-}\left(m_{1}, m_{2}\right)$ and $m \in \mathcal{E}^{+}\left(m_{1}, m_{2}\right)$. Inversely, let $m \in \mathcal{E}^{-}\left(m_{1}\right.$, $\left.m_{2}\right) \cap \mathcal{E}^{+}\left(m_{1}, m_{2}\right)$; then $\mathcal{R}\left(m_{1}, m, m_{2}\right)=m$ and so $m$ is the unique $\left(m_{1}, m_{2}\right)$-stabilizable mean.

Now, we are in a position to state the next result ensuring the existence of a maximal super-stabilizable (resp. minimal sub-stabilizable) mean.

Theorem 3.2 Let $m_{1}, m_{2}$ be two symmetric monotone means. Then the set $\mathcal{E}^{+}\left(m_{1}, m_{2}\right)$ has at least a maximal element.

Before giving the proof of the last theorem we state the next corollary, which is immediate from the above.

Corollary 3.3 Let $m_{1}, m_{2}$ be as in the above theorem. Then the set $\mathcal{E}^{-}\left(m_{1}, m_{2}\right)$ has at least a minimal element.

Proof For proving the theorem, we will show that the set $\mathcal{E}^{+}\left(m_{1}, m_{2}\right)$ is (nonempty) inductively ordered. Let us equip $\mathcal{E}^{+}\left(m_{1}, m_{2}\right)$ with the point-wise order induced by that of the set 
of all means. Let $E \subset \mathcal{E}^{+}\left(m_{1}, m_{2}\right)$ be a nonempty total ordered set and we get $E=\left(m_{i}\right)_{i \in J}$. Then, $\sup _{i \in J} m_{i}$ is a mean. Clearly, $\sup _{i \in J} m_{i}$ is an upper bound of $E$ and we wish to establish that $\sup _{i \in J} m_{i} \in \mathcal{E}^{+}\left(m_{1}, m_{2}\right)$. Indeed, for all $i \in J$, we have

$$
m_{i} \in E \quad \Longrightarrow \quad m_{i} \in \mathcal{E}^{+}\left(m_{1}, m_{2}\right) \quad \Longrightarrow \quad m_{i} \leq \mathcal{R}\left(m_{1}, m_{i}, m_{2}\right)
$$

Since $m_{1}$ and $m_{1}$ are monotone, we deduce by Theorem 2.1, $m_{i} \leq \mathcal{R}\left(m_{1}, \sup _{i \in J} m_{i}, m_{2}\right)$ for all $i \in J$ and so $\sup _{i \in J} m_{i} \leq \mathcal{R}\left(m_{1}, \sup _{i \in J} m_{i}, m_{2}\right)$, that is, $\sup _{i \in J} m_{i} \in \mathcal{E}^{+}\left(m_{1}, m_{2}\right)$. It follows that every nonempty totally ordered subset of $\mathcal{E}^{+}\left(m_{1}, m_{2}\right)$ has an upper bound in $\mathcal{E}^{+}\left(m_{1}, m_{2}\right)$, that is, $\mathcal{E}^{+}\left(m_{1}, m_{2}\right)$ is inductive. We can then apply the classical Zorn lemma to conclude and the proof of the theorem is complete.

Remark 3.1 A question arises from the above: Let $m_{1}$ and $m_{2}$ be two given symmetric means. Is it true that

$$
\mathcal{E}^{+}\left(m_{1}, m_{2}\right) \cup \mathcal{E}^{-}\left(m_{1}, m_{2}\right)=\mathcal{M}_{s} ?
$$

Proposition 3.4 For all given symmetric mean $m$, we have:

(1) The sets $\mathcal{E}^{-}(A, m)$ and $\mathcal{E}^{+}(A, m)$ are (linearly) convex.

(2) The sets $\mathcal{E}^{-}(G, m)$ and $\mathcal{E}^{+}(G, m)$ are geometrically convex.

Proof (1) follows from the linear-affine character of $A$ with the definition of $\mathcal{R}$, while (2) comes from the geometric character of $G$. The details are simple and omitted here.

\section{Sub-stabilizability and super-stabilizability}

The next definition may be stated.

Definition 4.1 Let $m_{1}, m_{2}$ be two nontrivial stable comparable means. A mean $m$ is called:

(a) ( $\left.m_{1}, m_{2}\right)$-sub-stabilizable if $\mathcal{R}\left(m_{1}, m, m_{2}\right) \leq m$ and $m$ is between $m_{1}$ and $m_{2}$,

(b) $\left(m_{1}, m_{2}\right)$-super-stabilizable if $m \leq \mathcal{R}\left(m_{1}, m, m_{2}\right)$ and $m$ is between $m_{1}$ and $m_{2}$.

Following Theorem 2.3, the above definition extends that of stabilizability in the sense that a mean $m$ is $\left(m_{1}, m_{2}\right)$-stabilizable if and only if (a) and (b) hold. It follows that the above concepts bring something new for non-stable and non-stabilizable means. For this, we say that $m$ is strictly $\left(m_{1}, m_{2}\right)$-sub-stabilizable if $\mathcal{R}\left(m_{1}, m, m_{2}\right)<m$ and $m$ is strictly $\left(m_{1}, m_{2}\right)$ super-stabilizable if $m<\mathcal{R}\left(m_{1}, m, m_{2}\right)$, with in both cases $m$ being strictly between $m_{1}$ and $m_{2}$.

With the notation of the above section we have

$$
\begin{aligned}
& m \text { is }\left(m_{1}, m_{2}\right) \text {-sub-stabilizable } \quad \Longrightarrow m \in \mathcal{E}^{-}\left(m_{1}, m_{2}\right), \\
& m \text { is }\left(m_{1}, m_{2}\right) \text {-super-stabilizable } \quad \Longrightarrow \quad m \in \mathcal{E}^{+}\left(m_{1}, m_{2}\right)
\end{aligned}
$$

and

$$
m \text { is }\left(m_{1}, m_{2}\right) \text {-sub-stabilizable } \Longleftrightarrow m^{*} \text { is }\left(m_{1}^{*}, m_{2}^{*}\right) \text {-super-stabilizable. }
$$

Example 4.1 We can easily see that $G$ is $(G, A)$-super-stabilizable (but not strictly) while $A$ is $(G, A)$-sub-stabilizable. However, $T$ and $M$ are not $(G, A)$-sub-stabilizable, since they 
are not between $G$ and $A$. More interesting examples, presented as main results, will be stated in the section below.

Theorem 4.1 Let $m$ be a continuous symmetric mean. Then the following assertions are met:

(1) If there exists a symmetric mean $m_{1}$ such that $m$ is $\left(m_{1}, G\right)$-sub-stabilizable then $m \geq m_{1}^{\pi}$.

(2) If there exists a symmetric mean $m_{1}$ such that $m$ is $\left(m_{1}, G\right)$-super-stabilizable then $m \leq m_{1}^{\pi}$.

Proof (1) Assume that $m$ is $m_{1}$-sub-stabilizable, that is,

$$
\forall a, b>0, \quad \mathcal{R}\left(m_{1}, m, G\right)(a, b) \leq m(a, b)
$$

or, according to the definition of $\mathcal{R}$,

$$
\forall a, b>0, \quad m_{1}(\sqrt{a}, \sqrt{b}) m(\sqrt{a}, \sqrt{b}) \leq m(a, b) .
$$

This, with a simple mathematical induction, implies that the inequality

$$
\forall a, b>0, \quad \prod_{n=1}^{N} m_{1}\left(a^{1 / 2^{n}}, b^{1 / 2^{n}}\right) m\left(a^{1 / 2^{N}}, b^{1 / 2^{N}}\right) \leq m(a, b)
$$

holds true for each integer $N \geq 1$. Letting $N \rightarrow \infty$ in the latter inequality and using the fact that $m$ is continuous we infer that

$$
\prod_{n=1}^{\infty} m_{1}\left(a^{1 / 2^{n}}, b^{1 / 2^{n}}\right) \leq m(a, b)
$$

which with equation (2.2) means that $m \geq m_{1}^{\pi}$.

(2) It is similar to that the above. The details are omitted here.

The above theorem has various consequences, which we will state in what follows.

Corollary 4.2 Let $m$ be a continuous symmetric mean. Then the next statements hold true:

(i) If $m$ is $\left(B_{p}, G\right)$-sub-stabilizable for some $p \geq 0$ then $L_{p} \leq m \leq B_{p}$. In particular, if $m$ is $(A, G)$-sub-stabilizable then $L \leq m \leq A$.

(ii) If $m$ is $\left(B_{p}, G\right)$-super-stabilizable for some $p \leq 0$ then $B_{p} \leq m \leq L_{p}$. In particular, if $m$ is $(A, G)$-super-stabilizable then $G \leq m \leq L$.

Proof It is immediate by combining the above theorem with the fact that $B_{p}^{\pi}=L_{p}$ for each real number $p$, and $B_{1}=A, L_{1}=L$.

Remark 4.1 (i) The above corollary tells us that $L$ is a minimal element of $\mathcal{E}^{-}(A, G)$ and it is a maximal element of $\mathcal{E}^{+}(A, G)$ : this rejoins the fact that $L$ is $(A, G)$-stabilizable.

(ii) The above corollary implies that $I$ is not $(A, G)$-super-stabilizable, but it is perhaps $(A, G)$-sub-stabilizable. See more details as regards the latter point in the section below. 
Corollary 4.3 Let $m>G$ be a strictly $\left(B_{p}, G\right)$-sub-stabilizable mean. Then $0<q<p<r$, where $q$ is the greatest number such that $m>L_{q}$ and $r$ is the smallest number such that $m<B_{r}$.

Proof If $m>G$ is strictly $\left(B_{p}, G\right)$-sub-stabilizable then, by definition, $m<B_{p}$ and, by the above corollary, $m \geq L_{p}$. Combining these latter mean-inequalities we deduce the desired result.

Corollary 4.4 (i) If there exists $p$ such that $P$ is strictly $\left(B_{p}, G\right)$-sub-stabilizable then $2 / 3<$ $p \leq 2$.

(ii) If $M$ is strictly $\left(B_{p}, G\right)$-sub-stabilizable for some $p$ then $4 / 3<p \leq 4$.

(iii) If $T$ is strictly $\left(B_{p}, G\right)$-sub-stabilizable then $5 / 3<p \leq 5$.

(iv) There is no $p \in \mathbb{R}$ such that $P, M$ or $T$ is $\left(B_{p}, G\right)$-super-stabilizable.

Proof Combining the above corollary with Theorem 1.1, we immediately deduce the assertions (i), (ii), and (iii).

Assertion (iv) follows from Corollary 4.2(ii) with Theorem 1.1 again. Details are omitted here.

\section{Application to some standard means}

This section will be devoted to an application of the above concepts to some known means. We begin with the next result.

Theorem 5.1 The logarithmic mean L is strictly $(G, A)$-super-stabilizable.

Proof First, the reader will do well to distinguish between the two next statements: ' $L$ is strictly $(G, A)$-super-stabilizable' to prove here and ' $L$ is $(A, G)$-stabilizable' already shown in [11]. By definition and by a simple reduction, we have to prove

$$
(L(a, b))^{2}<L\left(a, \frac{a+b}{2}\right) L\left(\frac{a+b}{2}, b\right)
$$

for all $a, b>0$ with $a \neq b$. We will present two different proofs for equation (5.1). By the symmetric character of the involved means, we can assume, without loss the generality, that $a<b$.

- The first method is much more natural: Since $A-a=b-A=(b-a) / 2$, we have

$$
L(a, A) L(A, b)=\frac{(b-a)^{2}}{4 \ln (A / a) \cdot \ln (b / A)} .
$$

Then by the inequality

$$
x y<\left(\frac{x+y}{2}\right)^{2}
$$

valid for all real numbers $x, y$ with $x \neq y$, one has

$$
4 \ln (A / a) \cdot \ln (b / A)<(\ln (A / a)+\ln (b / A))^{2}=(\ln (a / b))^{2} .
$$

This gives equation (5.1), so it completes the proof of the first method. 
- The second method is based on the fact that we can always set $a=e^{-x} G$ and $b=e^{x} G$ with $x>0$. A simple computation leads to

$$
L(a, b)=\frac{\operatorname{sh} x}{x} G, \quad L\left(a, \frac{a+b}{2}\right)=\frac{\operatorname{sh} x}{x-\ln (\operatorname{ch} x)} G, \quad L\left(a, \frac{a+b}{2}\right)=\frac{\operatorname{sh} x}{x+\ln (\operatorname{ch} x)} G .
$$

Substituting these in equation (5.1) we are in a position to show that

$$
\frac{\operatorname{sh}^{2} x}{x^{2}}<\frac{\operatorname{sh}^{2} x}{x^{2}-(\ln (\operatorname{ch} x))^{2}}
$$

for all $x>0$, which clearly holds and inequality (5.1) is again proved.

In summary, we have shown that $L$ is strictly $(G, A)$-super-stabilizable.

Remark 5.1 We can also see that $L$ is strictly $(A, H)$-sub-stabilizable. In fact, since $L$ is $(A, G)$-stabilizable and $G>H$, we obtain (with the help of Theorem 2.1)

$$
L=\mathcal{R}(A, L, G)>\mathcal{R}(A, L, H)
$$

which, with $H<L<A$, means that $L$ is strictly $(A, H)$-sub-stabilizable.

Theorem 5.2 The identric mean I is strictly $(A, G)$-sub-stabilizable.

Proof We will present here two different methods for proving our claim: The first is direct and based on some mean-inequalities already stated in the literature, while the second one is similar to above.

- First method: We have to show

$$
I(a, G)+I(b, G)<2 I(a, b)
$$

for all $a, b>0$ with $a \neq b$. If we recall that [15] the function $(x, y) \longmapsto I(x, y)$ is concave upon both variables, we immediately deduce that

$$
2 I(A, G)>I(a, G)+I(b, G) .
$$

Otherwise, it is well known that $\frac{A+G}{2}<I$ (see [13] for example) and $I(a, b)<A(a, b):=\frac{a+b}{2}$ for all $a, b>0, a \neq b$. We then obtain

$$
I(A, G)<\frac{A+G}{2}<I
$$

which, when combined with equation (5.3), gives equation (5.2), so it completes the proof of the first method.

- Second method: To show equation (5.2) is equivalent to proving that

$$
A(\sqrt{a}, \sqrt{b}) I(\sqrt{a}, \sqrt{b})<I(a, b) .
$$

As previously, we can easily verify that

$$
I(a, b)=G \exp \frac{x}{\operatorname{th} x}, \quad A(\sqrt{a}, \sqrt{b})=G^{1 / 2} \operatorname{ch}(x / 2) .
$$


Substituting these in the above and using the identity

$$
\operatorname{th} x=\frac{2 \operatorname{th}(x / 2)}{1+\operatorname{th}^{2}(x / 2)}
$$

valid for each $x>0$, the desired inequality is reduced to showing that

$$
\Phi(x):=\ln (\operatorname{ch}(x / 2))-(x / 2) \operatorname{th}(x / 2)<0
$$

for all $x>0$. A simple computation leads to

$$
\Phi^{\prime}(x)=-\frac{x}{4 \operatorname{ch}^{2}(x / 2)}<0
$$

It follows that $\Phi$ is strictly decreasing for $x>0$ and so $\Phi(x)<\Phi(0):=\lim _{t \rightarrow 0} \Phi(t)=0$. The second method is complete.

Remark 5.2 Another method for proving equation (5.4) can be stated as follows: It is well known (and easy to verify) that $I\left(a^{2}, b^{2}\right)=I(a, b) S(a, b)$ for all $a, b>0$, where $S:=$ $S(a, b)=\left(a^{a} b^{b}\right)^{1 /(a+b)}$ is the so-called weighted geometric mean. With this, equation (5.4) is equivalent to $A(\sqrt{a}, \sqrt{b})<S(\sqrt{a}, \sqrt{b})$ i.e. $A<S$, which is a well-known mean-inequality.

As a consequence of the above, the next result gives a double inequality refining $L<I$ and involving the four standard means $G, L, I$, and $A$.

\section{Corollary 5.3 We have}

$$
2 e^{2} L^{2}<G(A+G) \exp \frac{A+G}{L}<2 e^{2} I^{2} .
$$

Proof The above theorem means that $\mathcal{R}(A, I, G)<I$, which, with Theorem 2.1 and the fact that $L$ is $(A, G)$-stabilizable, yields

$$
L=\mathcal{R}(A, L, G)<\mathcal{R}(A, I, G)<I .
$$

This, with Example 2.1 and a simple manipulation, gives the desired result.

Of course, the above theorems when combined with the properties of sub-superstabilizability imply that $L^{*}$ is, simultaneously, strictly $(G, H)$-sub-stabilizable and strictly $(H, A)$-super-stabilizable, while $I^{*}$ is strictly $(H, G)$-super-stabilizable.

As already pointed out before, whether the first Seiffert mean $P$ is stabilizable still is an open problem. However, the next result may be stated.

Theorem 5.4 The first Seiffert mean P is strictly $(A, G)$-sub-stabilizable.

Proof Explicitly, we have to prove that

$$
A(\sqrt{a}, \sqrt{b}) P(\sqrt{a}, \sqrt{b})<P(a, b)
$$

holds for all $a, b>0$ with $a \neq b$. We also present here two different methods. 
- First method: this method is analogous to the above. Simple computation leads to

$$
P(a, b)=G \frac{\operatorname{sh} x}{\arcsin (\operatorname{th} x)}
$$

for each $x>0$. After simple substitution and reduction we are in a position to show that

$$
\Phi(x):=2 \arcsin (\operatorname{th}(x / 2))-\arcsin (\operatorname{th} x)>0
$$

for every $x>0$. We can easily obtain (after computation and reduction)

$$
\Phi^{\prime}(x)=\frac{1}{\operatorname{ch}(x / 2)}-\frac{1}{\operatorname{ch} x}>0
$$

for all $x>0$. The desired inequality follows in the same way as previously.

- Second method: this method is based on an integral form of $P(a, b)$. It is easy to see that, for all $a, b>0$ (with $a<b$ without loss the generality), we have

$$
P(a, b)=\left(\frac{4}{b-a} \int_{1}^{\sqrt{b / a}} \frac{d x}{1+x^{2}}\right)^{-1}
$$

This, with a simple manipulation, yields

$$
A(\sqrt{a}, \sqrt{b}) P(\sqrt{a}, \sqrt{b})=\left(\frac{8}{b-a} \int_{1}^{\sqrt[4]{b / a}} \frac{d x}{1+x^{2}}\right)^{-1} .
$$

To show equation (5.6) is equivalent to proving that the second side of equation (5.8) is strictly smaller than that of equation (5.7), or again (after a simple reduction)

$$
\int_{1}^{\sqrt{b / a}} \frac{d x}{1+x^{2}}<2 \int_{1}^{\sqrt[4]{b / a}} \frac{d x}{1+x^{2}}
$$

If we use the variable of change $x=t^{2}, t>0$ in the left integral of equation (5.9) our aim is then reduced to showing that

$$
\int_{1}^{\sqrt[4]{b / a}} \frac{x d x}{1+x^{4}}<\int_{1}^{\sqrt[4]{b / a}} \frac{d x}{1+x^{2}}
$$

It is very easy to verify that

$$
\forall x>0, x \neq 1, \quad \frac{x}{1+x^{4}}<\frac{1}{1+x^{2}},
$$

from which equation (5.10) follows. The proof is complete.

Remark 5.3 Another way of proving equation (5.6) can be followed: For all $a, b>0, a \neq b$, we have [16]

$$
P\left(a^{2}, b^{2}\right)>(A(a, b))^{2}>(P(a, b))^{2} .
$$


This gives

$$
P\left(a^{2}, b^{2}\right)>(A(a, b))^{2}>P(a, b) A(a, b)
$$

which is exactly equation (5.6).

\section{Some open problems}

In the above section, we have proved that $P$ is strictly $(A, G)$-sub-stabilizable. The fact that $P$ is strictly $(G, A)$-super-stabilizable is not proved yet. This is equivalent to showing that

$$
(P(a, b))^{2}<P\left(a, \frac{a+b}{2}\right) P\left(\frac{a+b}{2}, b\right)
$$

holds for all $a, b>0$ with $a \neq b$. As above, and setting $t=\operatorname{th} x, x>0$, we are in a position to show that

$$
\Phi(t):=(\arcsin t)^{2}-4 \arcsin \frac{t}{2+t} \arcsin \frac{t}{2-t}>0
$$

for all $0<t<1$. We then present the following.

Problem 1: Prove or disprove that the first Seiffert mean $P$ is strictly $(G, A)$-superstabilizable.

Problem 2: Find the best real numbers $p>0$ and $q>0$ for which $P$ is strictly $\left(B_{p}, B_{q}\right)$ sub-stabilizable.

Problem 3: Are the means $T$ and $M$ strictly $\left(B_{p}, B_{q}\right)$-sub-stabilizable for some real numbers $p>0, q>0$ ?

\section{Competing interests}

The authors declare that they have no competing interests.

\section{Authors' contributions}

Both authors jointly worked, read and approved the final manuscript.

\section{Author details}

${ }^{1}$ Department of Mathematics, Science Faculty, Taibah University, P.O. Box 30097, Al Madinah Al Munawwarah, 41477. Kingdom of Saudi Arabia. ${ }^{2}$ Department of Mathematics, Science Faculty, Moulay Ismail University, Meknes, Morocco. ${ }^{3}$ Department of Mathematics, Babes-Bolyai University, Str. Kogalniceanu nr. 1, Cluj-Napoca, 400084, Romania.

\section{Acknowledgements}

The present work was supported by the Deanship of Scientific Research of Taibah University.

Received: 27 July 2013 Accepted: 18 December 2013 Published: 24 Jan 2014

\section{References}

1. Bullen, PS: Handbook of Means and Their Inequalities, 2nd edn. Mathematics and Its Applications. Springer, Berlin (1987)

2. Seiffert, HJ: Problem 887. Nieuw Arch. Wiskd. 11, 176 (1993)

3. Seiffert, HJ: Aufgabe 16. Wurzel 29, 87 (1995)

4. Neuman, E, Sándor, J: On the Schwab-Borchardt mean. Math. Pannon. 14(2), 253-266 (2003)

5. Raïssouli, M, Sándor, J: On a method of construction of new means with applications. J. Inequal. Appl. 2013, 89 (2013)

6. Chu, YM, Long, BY, Gong, WM, Song, YQ: Sharps bounds for Seiffert and Neuman-Sándor means in terms of generalized logarithmic means. J. Inequal. Appl. 2013, 10 (2013)

7. Chu, YM, Long, BY: Bounds of the Neuman-Sándor mean using power and identric means. Abstr. Appl. Anal. 2013, Article ID 832591 (2013)

8. Hästö, PA: Optimal inequalities between Seiffert's mean and power means. MIA Preprint (2013)

9. Yang, ZH: Sharp bounds of the second Seiffert mean in terms of power means (2012). arXiv:1206.5494v1 [math.CA]

10. Yang, ZH: Sharp power means bounds for Neuman-Sándor mean (2012). arXiv:1208.0895 [math.CA]

11. Raïssouli, M: Stability and stabilizability for means. Appl. Math. E-Notes 11, 159-174 (2011) 
12. Raïssouli, M: Stabilizability of the Stolarsky mean and its approximation in terms of the power binomial mean. Int. J. Math. Anal. 6(18), 871-881 (2012)

13. Raïssouli, M: Refinements for mean-inequalities via the stabilizability concept. J. Inequal. Appl. 2012,55 (2012)

14. Raïssouli, M: Positive answer for a conjecture about stabilizable means. J. Inequal. Appl. 2013, 467 (2013)

15. Sándor, J: Monotonicity and convexity properties of means. Octogon Math. Mag. 7(2), 22-27 (1999)

16. Sándor, J: On certain inequalities for means, III. Arch. Math. 76, 34-40 (2001)

10.1186/1029-242X-2014-28

Cite this article as: Raïssouli and Sándor: Sub-stabilizability and super-stabilizability for bivariate means. Journal of Inequalities and Applications 2014, 2014:28

Submit your manuscript to a SpringerOpen ${ }^{\circ}$ journal and benefit from:

- Convenient online submission

- Rigorous peer review

- Immediate publication on acceptance

- Open access: articles freely available online

- High visibility within the field

- Retaining the copyright to your article 